\title{
Image processing in life science. Applications from cells to food.
}

\author{
F. Perez Alvarado, M.A. Hussein, T. Becker. \\ Group of (Bio-) process analysis and technology, \\ Center of Life Science Weihenstephan, \\ Technische Universität München, Freising, Weihenstephaner Steig 20
}

\begin{abstract}
:
Several problems are encountered in modeling and processing of many life science applications such like tissue engineering, bioacoustics, and food processing detection. Recently, various image processing techniques have been developed in an attempt to solve these problems. Due to the rapid advance and high number of researches included in such field, there is always a room for improvement. In this work, advanced image processing approaches are presented and applied to different life science applications. The first approach utilizes image processing in the generation of micro-scaffolds for tissue engineering modeling. Furthermore, a spectrogram enhancement approach is developed to improve the pattern detection of weevils existence in grain storage, based on their detected and identified sounds. Finally, a novel edge detection method applied on intramuscular fat recognition is presented based on the energy and skewness as two smoothed versions of the meat image. Results, in comparison to experimental data, offer a maximum error of $7.81 \%$ in the specified detection process.
\end{abstract}

\section{Material and Method}

\subsection{Generation of micro-porous approach}

The difficulty of modeling such complex micro-structures always lies in retrieving the geometry into the solver as edges. Edge detection algorithms are based upon highlighting discontinuities in images and were first developed for satellite images. The technique has become well-known and was further developed and widely used for other image processing applications. During the conventional detection procedures, usually all the image size is processed, which leads to redundant calculations of areas containing no edge information. In this study a redundant avoiding algorithm is developed which searches for the threshold value in the image and then propagates around it in a 2-D lattice in eight directions figure 1.1, until an edge is hit.
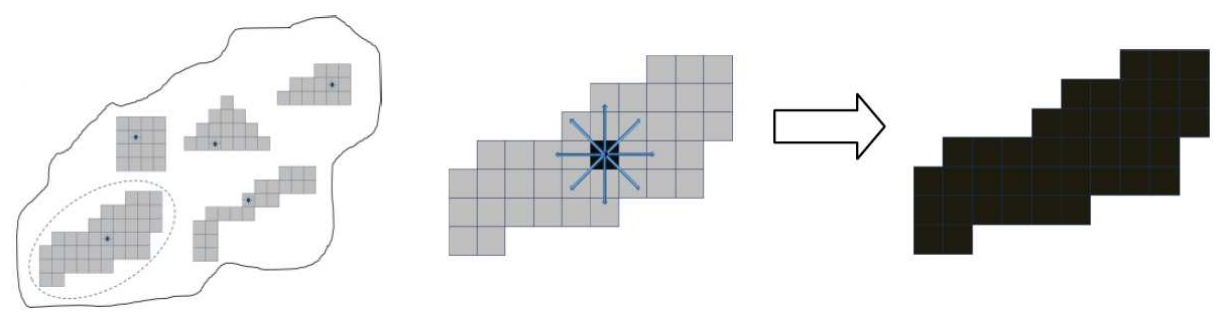

Figure 1.1 The scanning procedure for gradient changes propagates in an eight directional lattice until an edge is hit

\subsection{Spectrogram enhancement in bioacoustics}

This approach is based on detection of the sound pattern edges in the crest factor image, as shown in figure 1.2 and 1.3, which behaves as a smoothed version of the spectrogram image and avoids the use of edge detectors prior smoothing filters and their scaling constraints. 


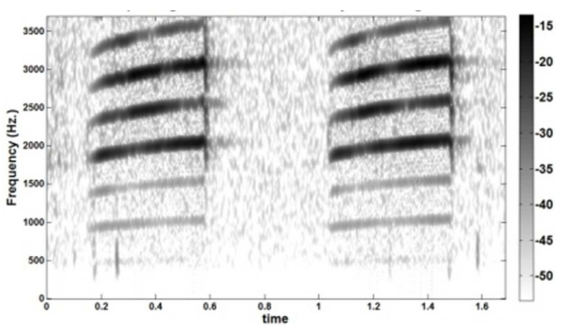

Figure 1.2 Spectrogram with $40 \mathrm{~dB}$ limited dynamic range
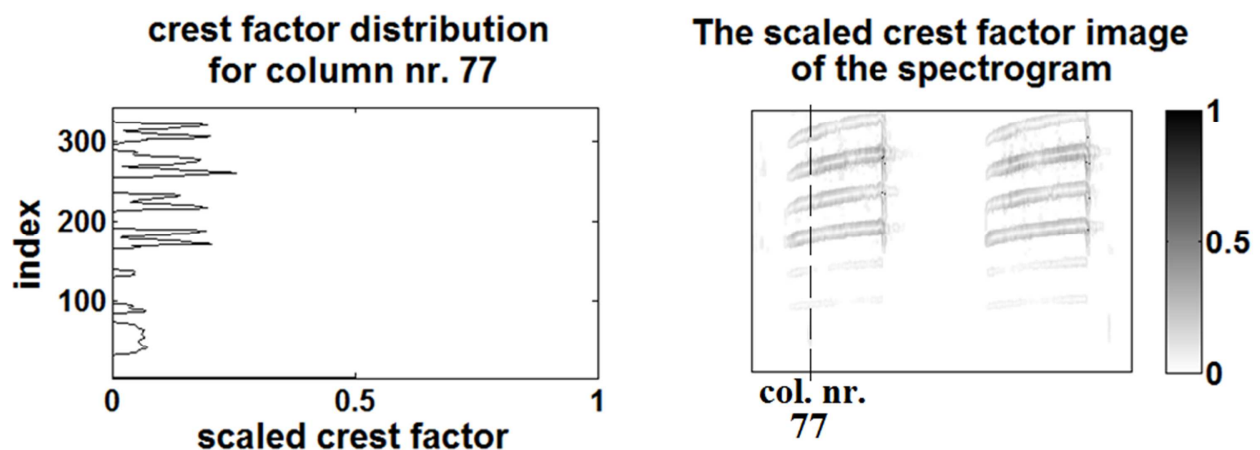

Figure 1.3 Crest factor distributions (left), the scaled crest factor image of the spectrogram (right).

\subsection{Edge detection for intramuscular fat recognition}

A novel edge detection method applied on intramuscular fat is presented based on the energy and skewness as two smoothed versions of the meat image, as shown in the flow chart given in figure 1.4.

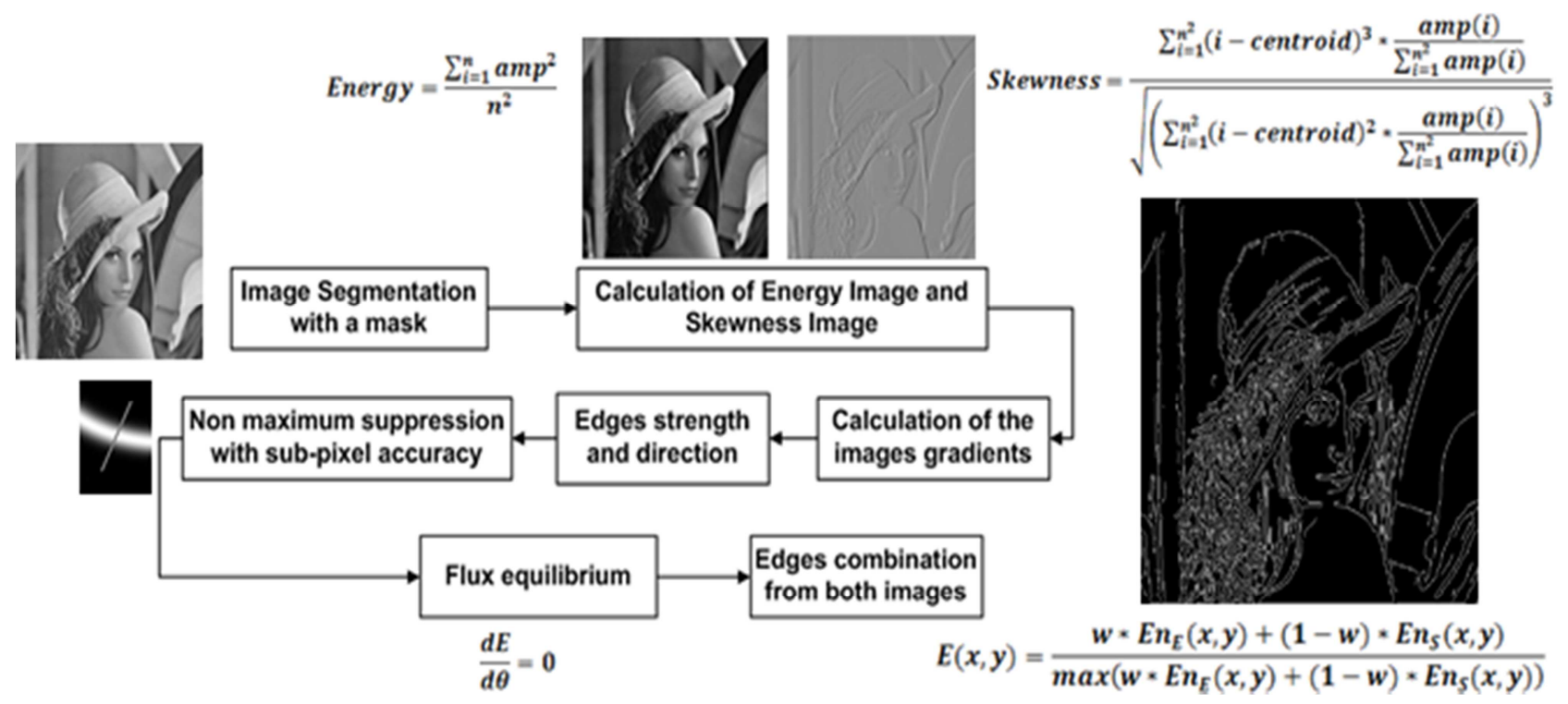

Figure 1.4 flow chart edge detection 


\section{Results}

\subsection{Generation of micro-porous Approach}

The conventional approach for edge detection is based upon either thresholding the first derivative (gradient) of the image or locating zero crossings of its second derivative (Laplacian).The sample used, figure 2.1, for this simulation was of size $3 \mathrm{~mm}, 350 \mu \mathrm{m}$ and $350 \mu \mathrm{m}$ in z-direction, x-direction and y-direction, respectively. The geometry was uploaded on the solver by collective segmenting and edge detection techniques. The method has proven time saving and as well enables the possibility of parallel processing of the image, as each threshold value can be scanned and dealt with independently. After the predictions of the edges, the 2-d binary edge images are assembled forming a 3-D binary file of the scaffolds figure 2.1.
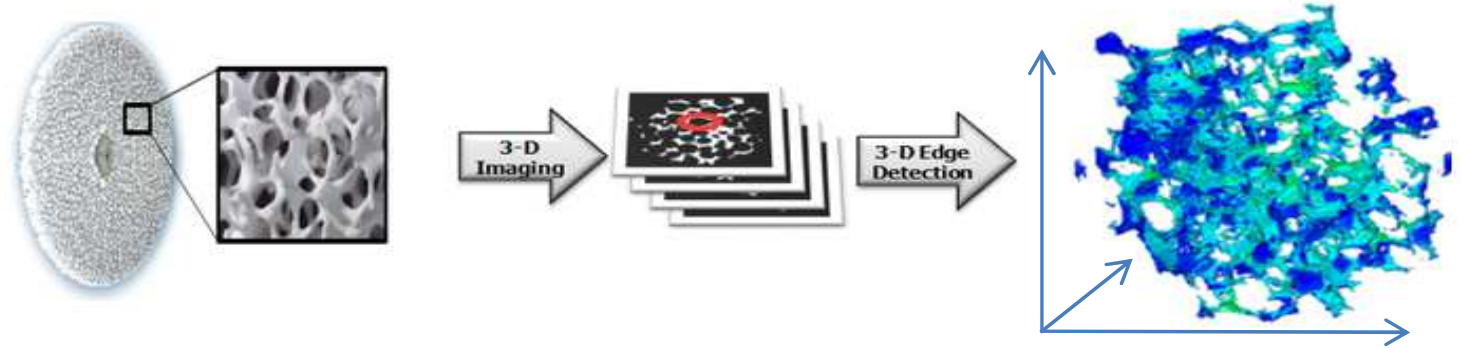

Figure 2.1 From the micro-CT images 430 hundred 2-D binary edge images are assembled forming a 3-D binary file of the scaffolds

\subsection{Spectrogram enhancement in bioacoustics}

The spectrogram enhancement approach is compared to other enhancement approaches, including multiband spectral subtraction and wavelet packet decomposition, for different structure bioacoustics calls including bats (ultrasound range) and birds (acoustic range), with additive white Gaussian noise added across a range of signal to noise ratios. Results measured subjectively and objectively, indicate that the proposed method is promising for efficiently spectrogram enhancing while preserving its temporal and spectral accuracy, as shown in figure 2.2.

(a)

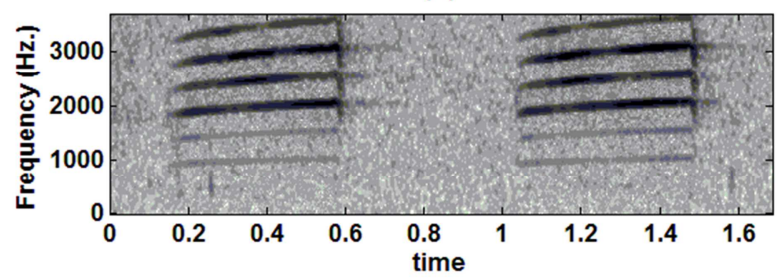

(c)

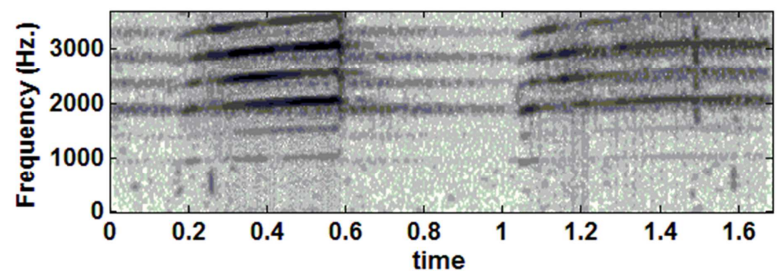

(e)

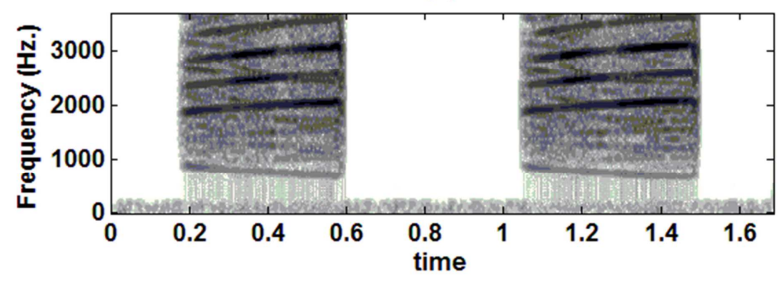

(b)

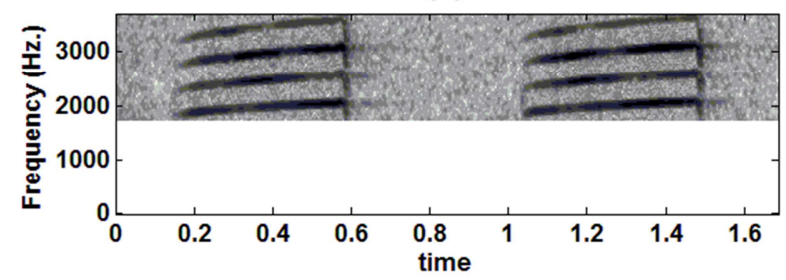

(d)

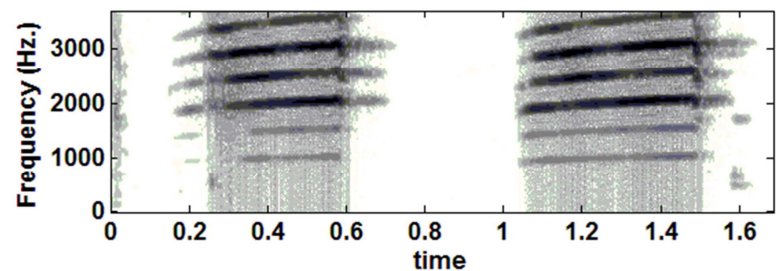

(f)

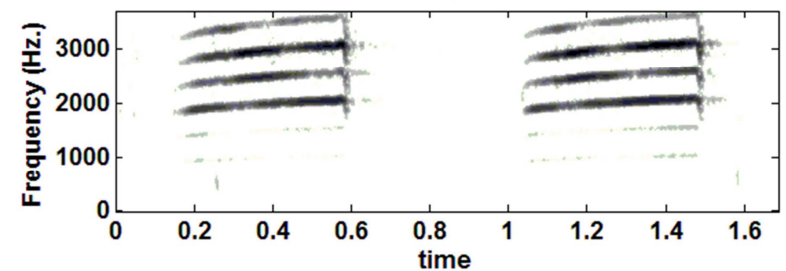

Figure 2.2 (a) One of Sitta Canadensis bird calls at SNR $=20 \mathrm{~dB}$, and its enhanced spectrogram by (b) BP, (c) MBSS, (d) WF, (e) WPD, and (f) the proposed method approaches. 


\subsection{Edge detection for intramuscular fat recognition}

The generation of micro-scaffolds approach is applied on a sample meat image, which is scanned line by line and every noticeable variance in the color values or the grey levels is captured and the local thresholds values are estimated according to the highest drop gradient value. The local threshold values are averaged to estimate the global threshold for the whole image, as shown in figure 2.1.

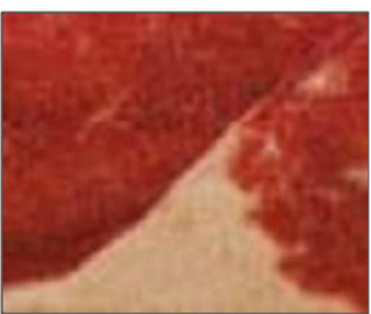

(a)

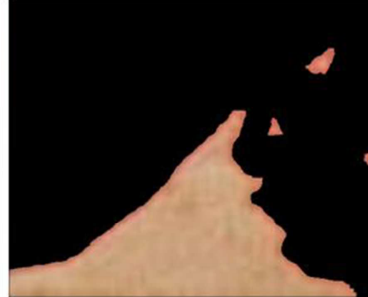

(b)

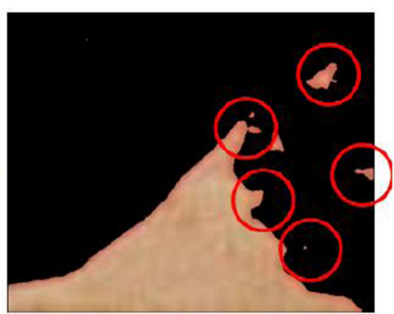

(c)

Figure 2.3 an image for a meat with fat (a), the $x$ y scan of the image which does not show all the fat details (b) and the lattice scan of the image showing more details of the fat, as circled by red (c).

For intramuscular fat, the developed edge detection approach is used to predict the fat content in marbled meat images, as shown in figure 2.3 .
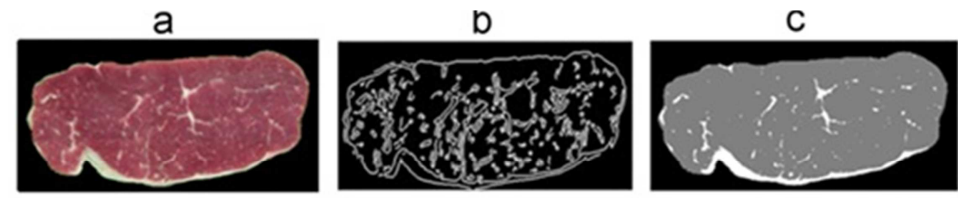

$7.2 \%$
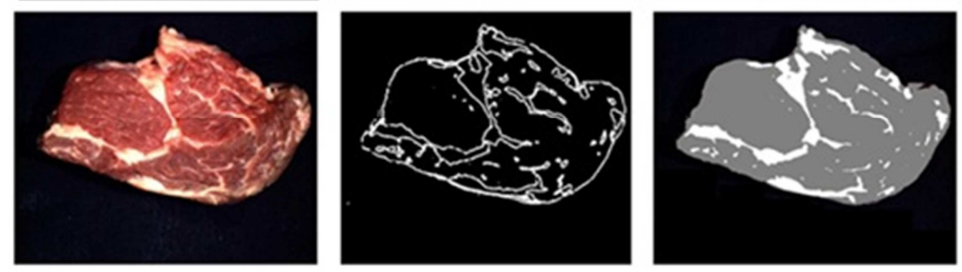

$18.5 \%$

Figure 2.4 (a) original marbled meat images, (b) edges images using the proposed method (c) detected fat regions, and (d) the percentage of fat content

\section{Conclusion}

As observed in the different approaches there is always a room for improvement that minimize the error observed in the specified detection process. The methods have proven time saving and as well enables the possibility of parallel processing of the image that leads to a more efficient performance highly desirable for industrial applications.

\section{References}

[1] Hussein, M. A., Becker, T.: Numerical Modelling of Shear and Normal Stress of Micro-Porous Ceramics for Stimulated in-vitro Cultivation of Bone Cells. Microfluidics and Nanofluidics 8 (2010), 665-675. DOI:10.1007/s10404-009-0499-1

[2] Hussein, W. B., Hussein, M. A., Becker, T.: Detection of the Red Palm Weevil Using Its Bioacoustics Features. Journal of Bioacoustics 19 (2010), 177-194.

[3] Hussein, W. B., Moaty, A. A, Hussein, M. A., Becker, T.: "A novel edge detection method with application to the fat content prediction in marbled meat". Pattern recognition (2011), doi:10.1016/j.patcog.2011.04.028 